\title{
On Neumann and Poincare problems for Laplace equation
}

\author{
VLADIMIR RYAZANOV
}

\begin{abstract}
It is proved the existence of nonclassical solutions of the Neumann problem for the harmonic functions in the Jordan rectifiable domains with arbitrary measurable boundary distributions of normal derivatives. The same is stated for a special case of the Poincare problem on directional derivatives. Moreover, it is shown that the spaces of the found solutions have the infinite dimension.
\end{abstract}

Key words and phrases : Neumann and Poincare problems, Laplace equation, harmonic functions, directional derivatives, nontangential limits.

Mathematics Subject Classification (2010) : primary 31A05, 31A20, 31A25, 31B25, 35Q15; secondary 30E25, 31C05, 34M50, 35F45.

\section{Introduction}

It is well-known that the Neumann problem has no classical solutions generally speaking even for some continuous boundary data. The main goal of this short note is to show that the problem has nonclassical solutions for arbitrary measurable data. The result is based on a reduction of this problem to the Hilbert (Riemann-Hilbert) boundary value problem recently solved for arbitrary measurable coefficients and for arbitrary measurable boundary data in [1, see also [2] on the Dirichlet problem.

Let us start from a more general problem on directional derivatives in the unit disk $\mathbb{D}=\{z \in \mathbb{C}:|z|<1\}, z=x+i y$. The classic setting of the latter problem is to find a function $u: \mathbb{D} \rightarrow \mathbb{R}$ that is twice continuously differentiable, admits a continuous extension to the boundary of $\mathbb{D}$ together with its first partial derivatives, satisfies the Laplace equation

$$
\Delta u:=\frac{\partial^{2} u}{\partial x^{2}}+\frac{\partial^{2} u}{\partial y^{2}}=0 \quad \forall z \in \mathbb{D}
$$

and the boundary condition with a prescribed continuous date $\varphi: \partial \mathbb{D} \rightarrow \mathbb{R}$ :

$$
\frac{\partial u}{\partial \nu}=\varphi(\zeta) \quad \forall \zeta \in \partial \mathbb{D}
$$


where $\frac{\partial u}{\partial \nu}$ denotes the derivative of $u$ at $\zeta$ in a direction $\nu=\nu(\zeta),|\nu(\zeta)|=1$ :

$$
\frac{\partial u}{\partial \nu}:=\lim _{t \rightarrow 0} \frac{u(\zeta+t \cdot \nu)-u(\zeta)}{t}
$$

The Neumann problem is a special case of the above problem on directional derivatives with the boundary condition

$$
\frac{\partial u}{\partial n}=\varphi(\zeta) \quad \forall \zeta \in \partial \mathbb{D}
$$

where $n$ denotes the unit interior normal to $\partial \mathbb{D}$ at the point $\zeta$.

In turn, the above problem on directional derivatives is a special case of the Poincare problem with the boundary condition

$$
a \cdot u+b \cdot \frac{\partial u}{\partial \nu}=\varphi(\zeta) \quad \forall \zeta \in \partial \mathbb{D}
$$

where $a=a(\zeta)$ and $b=b(\zeta)$ are real-valued functions given on $\partial \mathbb{D}$.

Recall also that twice continuously differentiable solutions of the Laplace equation are called harmonic functions. As well known, such functions are infinitely differentiable.

\section{On nonclassical solutions of boundary problems}

Let us start from the more general problem on directional derivatives.

Theorem 1. Let $\nu: \partial \mathbb{D} \rightarrow \mathbb{C},|\nu(\zeta)| \equiv 1$, and $\varphi: \partial \mathbb{D} \rightarrow \mathbb{R}$ be measurable functions. Then there exist harmonic functions $u: \mathbb{D} \rightarrow \mathbb{R}$ such that

$$
\lim _{z \rightarrow \zeta} \frac{\partial u}{\partial \nu}(z)=\varphi(\zeta)
$$

along any nontangential paths to a.e. point $\zeta \in \partial \mathbb{D}$.

Remark 1. We are able to say more in the case of $\operatorname{Re} n(\zeta) \cdot \overline{\nu(\zeta)}>0$. Indeed, the latter magnitude is a scalar product of $n=n(\zeta)$ and $\nu=\nu(\zeta)$ interpreted as vectors in $\mathbb{R}^{2}$ and it has the geometric sense of projection of the vector $\nu$ onto the inner normal $n$ to $\partial \mathbb{D}$ at the point $\zeta$. In view of (6), since the limit $\varphi(\zeta)$ is finite, there is a finite limit $u(\zeta)$ of $u(z)$ as $z \rightarrow \zeta$ in $\mathbb{D}$ along the straight line passing through the point $\zeta$ and being parallel to the vector $\nu$ because along this line

$$
u(z)=u\left(z_{0}\right)-\int_{0}^{1} \frac{\partial u}{\partial \nu}\left(z_{0}+\tau\left(z-z_{0}\right)\right) d \tau .
$$


Thus, at each point with condition (6) , there is the directional derivative

$$
\frac{\partial u}{\partial \nu}(\zeta):=\lim _{t \rightarrow 0} \frac{u(\zeta+t \cdot \nu)-u(\zeta)}{t}=\varphi(\zeta) .
$$

In particular, in the case of the Neumann problem, $\operatorname{Re} n(\zeta) \cdot \overline{\nu(\zeta)} \equiv 1>0$ and we have by Theorem 1 and Remark 1 the following significant result.

Theorem 2. For each measurable function $\varphi: \partial \mathbb{D} \rightarrow \mathbb{R}$, one can find harmonic functions $u: \mathbb{D} \rightarrow \mathbb{R}$ such that, at a.e. point $\zeta \in \partial \mathbb{D}$, there exist:

1) the finite radial limit

$$
u(\zeta):=\lim _{r \rightarrow 1} u(r \zeta)
$$

2) the normal derivative

$$
\frac{\partial u}{\partial n}(\zeta):=\lim _{t \rightarrow 0} \frac{u(\zeta+t \cdot n)-u(\zeta)}{t}=\varphi(\zeta)
$$

3) the nontangential limit

$$
\lim _{z \rightarrow \zeta} \frac{\partial u}{\partial n}(z)=\frac{\partial u}{\partial n}(\zeta)
$$

where $n=n(\zeta)$ denotes the unit interior normal to $\partial \mathbb{D}$ at the point $\zeta$.

Proof. To prove Theorem 1, let us show that the problem on directional derivatives is equivalent to the corresponding Riemann-Hilbert problem.

Indeed, let $u$ be a harmonic function $u: \mathbb{D} \rightarrow \mathbb{R}$ satisfying the boundary condition (6). Then the functions $U=u_{x}$ and $V=-u_{y}$ satisfy the system of Cauchy-Riemann: $U_{y}=-V_{x}$ and $U_{x}=V_{y}$ in view of (11). Thus, the function $f=U+i V$ is analytic in $\mathbb{D}$ and along any nontangential path to a.e. $\zeta \in \partial \mathbb{D}$

$$
\lim _{z \rightarrow \zeta} \operatorname{Re} \nu(\zeta) \cdot f(z)=\varphi(\zeta)
$$

that is equivalent to (6). Inversely, let $f: \mathbb{D} \rightarrow \mathbb{C}$ be an analytic function satisfying the boundary condition (12). Then any indefinite integral $F$ of $f$ is also a single-valued analytic function in $\mathbb{D}$ and $u=\operatorname{Re} F$ is a harmonic function satisfying the boundary condition (6) because the directional derivative

$$
\frac{\partial u}{\partial \nu}=\operatorname{Re} \bar{\nu} \cdot \nabla u=\operatorname{Re} \nu \cdot \overline{\nabla u}=(\nu, \nabla u)
$$

is the scalar product of $\nu$ and the gradient $\nabla u$ interpreted as vectors in $\mathbb{R}^{2}$.

Thus, Theorem 1 is a direct consequence of Theorem 2.1 in [1] on the Riemann-Hilbert problem with $\lambda(\zeta)=\overline{\nu(\zeta)}, \zeta \in \partial \mathbb{D}$. 
The following result in domains bounded by rectifiable Jordan curves is proved perfectly similar to Theorem 1 but it is based on more general Theorem 3.1 in [1].

Theorem 3. Let $D$ be a domain in $\mathbb{C}$ bounded by a rectifiable Jordan curve, $\nu: \partial D \rightarrow \mathbb{C},|\nu(\zeta)| \equiv 1$, and $\varphi: \partial D \rightarrow \mathbb{R}$ be measurable functions with respect to the natural parameter. Then there exist harmonic functions $u: D \rightarrow \mathbb{R}$ such that along any nontangential paths

$$
\lim _{z \rightarrow \zeta} \frac{\partial u}{\partial \nu}(z)=\varphi(\zeta)
$$

for a.e. point $\zeta \in \partial D$ with respect to the natural parameter.

Remark 2. Again we are able to say more in the case with $\operatorname{Re} n \cdot \bar{\nu}>0$ where $n=n(\zeta)$ is the unit inner normal at a point $\zeta \in \partial D$ with a tangent to $\partial D$. In view of (14), since the limit $\varphi(\zeta)$ is finite, there is a finite limit $u(\zeta)$ of $u(z)$ as $z \rightarrow \zeta$ in $\mathbb{D}$ along the straight line passing through the point $\zeta$ and being parallel to the vector $\nu$ because along this line, for $z$ and $z_{0}$ that are close enough to $\zeta$,

$$
u(z)=u\left(z_{0}\right)-\int_{0}^{1} \frac{\partial u}{\partial \nu}\left(z_{0}+\tau\left(z-z_{0}\right)\right) d \tau .
$$

Thus, at each point with the condition (14), there is the directional derivative

$$
\frac{\partial u}{\partial \nu}(\zeta):=\lim _{t \rightarrow 0} \frac{u(\zeta+t \cdot \nu)-u(\zeta)}{t}=\varphi(\zeta) .
$$

In particular, in the case of the Neumann problem, $\operatorname{Re} n(\zeta) \cdot \overline{\nu(\zeta)} \equiv 1>0$ and we have by Theorem 3 and Remark 2 the following significant result. Here we also apply the well-known fact that any rectifiable curve has a tangent a.e. with respect to the natural parameter.

Theorem 4. Let $D$ be a domain in $\mathbb{C}$ bounded by a rectifiable Jordan curve and $\varphi: \partial D \rightarrow \mathbb{R}$ be a measurable function with respect to the natural parameter. Then one can find harmonic functions $u: D \rightarrow \mathbb{R}$ such that, at a.e. point $\zeta \in \partial D$ with respect to the natural parameter, there exist:

1) the finite normal limit

$$
u(\zeta):=\lim _{z \rightarrow \zeta} u(z)
$$

2) the normal derivative

$$
\frac{\partial u}{\partial n}(\zeta):=\lim _{t \rightarrow 0} \frac{u(\zeta+t \cdot n)-u(\zeta)}{t}=\varphi(\zeta)
$$


3) the nontangential limit

$$
\lim _{z \rightarrow \zeta} \frac{\partial u}{\partial n}(z)=\frac{\partial u}{\partial n}(\zeta)
$$

where $n=n(\zeta)$ denotes the unit interior normal to $\partial D$ at the point $\zeta$.

Note that here the tangent $\tau(s)$ to $\partial D$ is measurable with respect to the natural parameter $s$ as the derivative $d \zeta(s) / d s$ and, thus, the inner normal $n(s)$ to $\partial D$ is also measurable with respect to the natural parameter.

Remark 3. Similarly, on the basis of Theorem 4.1 in [1], in the case of arbitrary Jordan domains $D$ in $\mathbb{C}$, we are able to conclude that there exist harmonic functions $u: D \rightarrow \mathbb{R}$ for which the limit relation (14) holds in the sense of the unique principal asymptotic value for a.e. $\zeta \in \partial D$ with respect to the harmonic measure, see the corresponding definitions and comments in [1], Section 4.

\section{On the dimension of spaces of solutions}

Finally, we have the following significant result.

Theorem 5. The spaces of harmonic functions in Theorems 1-4, being nonclassical solutions of the problem on directional derivatives and the Neumann problem, have the infinite dimension for any prescribed measurable boundary data.

Proof. In view of the equivalence of the problem on the directional derivatives to the corresponding Hilbert (Riemann-Hilbert) boundary value problem established under the proof of Theorem 1, the conclusion of Theorem 5 follows directly from Theorem 5.2 and Remark 5.2 in [1].

\section{References}

[1] V. Ryazanov, On the Riemann-Hilbert Problem without Index, Ann. Univ. Bucharest, Ser. Math. 5 (LXIII), no. 1 (2014), 169-178.

[2] V. Ryazanov, Infinite dimension of solutions of the Dirichlet problem, Open Math. (the former Central European J. Math.) 13, no. 1 (2015), 348-350.

Vladimir Ryazanov

Institute of Applied Mathematics and Mechanics

of National Academy of Sciences of Ukraine,

19 gen. Batyuk Str., 84116 Slavyansk, UKRAINE

E-mail: vl_ryazanov@mail.ru 